\title{
HIGH THROUGHPUT, MULTI-CHANNEL, NANO-MECHANICAL MEASUREMENTS OF SINGLE PLATELET CONTRACTION AS A DIAGNOSTIC MICRODEVICE FOR CLOTTING AND BLEEDING DISORDERS
}

\author{
David R. Myers ${ }^{1,2}$, Yongzhi Qiu ${ }^{1,2}$, Ashley Carson Brown ${ }^{2}$, Yumiko Sakurai ${ }^{1,2}$, Reginald Tran ${ }^{1,2}$, \\ Byungwook Ahn ${ }^{1,2}$, Robert Mannino ${ }^{1,2}$, Meredith Fay ${ }^{1}$, Hunter Strauss ${ }^{2}$, Todd Sulchek ${ }^{2}$, \\ Andrew Lyon ${ }^{2}$, Thomas Barker ${ }^{2}$, Wilbur A. Lam ${ }^{1,2}$ \\ ${ }^{1}$ Emory University School of Medicine, Atlanta, Georgia, USA \\ ${ }^{2}$ Georgia Institute of Technology, Atlanta, Georgia, USA
}

\begin{abstract}
Platelet contraction, which occurs during blood clot formation, is highly variable and dependent on the local mechanical and biochemical environment. We present a novel device capable of measuring individual platelet contraction in a high throughput manner. Platelets adhere and contract on micronsized protein stamps patterned on polyacrylamide strips, which have tunable stiffness. Four polyacrylamide strips are placed in a device and each is encased in a separate microfluidic, enabling precise control of the biochemical and flow conditions around each platelet. Using this device, we demonstrate how forces applied by platelets change with differing biochemical agonists and substrate stiffness.
\end{abstract}

\section{INTRODUCTION}

\section{Clot mechanics and disease}

Blood clots are composed of fibrin, platelets, other blood cells, and proteins which interact to prevent hemorrhage (Figure 1). During clot formation, platelets apply tensile forces to the fibrin mesh, reducing its overall size and increasing the stiffness of the clot. Previous studies on clot formation have shown that the mechanical properties of clots are related to thrombotic or hemostatic diseases [1]. For example, clots are 50\% stiffer and more resistant to dissolution in young patients with postmyocardial infarction [2] than clots from healthy controls. In addition, mechanical properties of clots formed from peripheral blood are altered in patients who develop idiopathic thromboemboli or acute ischemic strokes compared to those from healthy controls $[3,4]$. As such, understanding clot mechanics is of vital importance to fundamentally understanding thrombosis and thromboembolism in cardiovascular disease and stroke.

However, there is currently no diagnostic test to assess platelet contraction force, especially at the single cell level and in the context of different mechanical environments, which has previously been demonstrated to significantly affect the platelet contraction force ${ }^{[5]}$. Furthermore, platelets have a wide variance in applied forces for a given mechanical and biochemical condition. As such, statistically significant results will require large numbers of measured platelets.

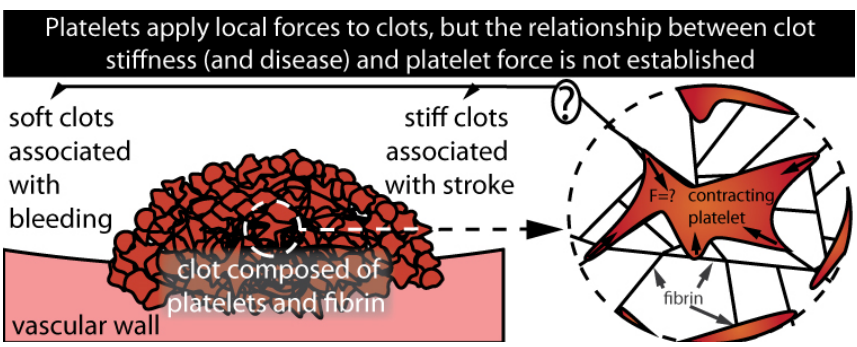

Figure 1: Individual platelet force varies and is dependent on environmental factors. To understand how platelet force correlates to clot stiffness, and thus pathological conditions, population data on platelet contraction in a variety of biophysical and biochemical conditions is needed.

\section{Previous work on platelet contraction}

Several methods have been used to measure platelet contraction at the single cell level using atomic force microscopy [5] or traction force microscopy [6]. Other groups have measured small aggregates of platelets using PDMS microposts [7]. The AFM experiments specifically varied the mechanical stiffness of the cantilever and also created an infinite stiffness condition, finding that platelets apply increasing force with increasing mechanical stiffness.

Alternatively, microposts have been used to measure small aggregates of platelets and have been proposed for measuring single platelet contraction. In this scheme, microposts are coated with a protein and platelets adhere to and move the top of the posts. Our calculations estimate the need for sub-micron microposts to measure single platelet contraction, which would be extremely difficult to fabricate and measure with precision. Another concern is that studies examining how platelets are affected by different stiffness conditions are difficult since changing the post stiffness requires changing the surface area exposed to the platelet. Since the proteins which the platelet adheres to during contraction also activate the platelet, any results derived using microposts are confounded by this effect.

A diagnostic capable of measuring the nano-mechanics of single platelet contraction in a high throughput manner can meet this clinical need and potentially revolutionize how we assess platelet function, and ultimately enable clinicians to better predict heart attacks and stroke.

\section{DESIGN CONSIDERATIONS}

One recent technique greatly reduced the computational and experimental constraints by creating arrays of fluorescently tagged protein dots on the surface of a polyacrylamide gel using lithographic techniques [8]. Cells adhere to a collection of dots and may move each dot independently. The cell may apply an independent traction force, $\boldsymbol{T}$, to each calculated as:

$$
\boldsymbol{T}=\frac{2 \pi G a u}{2-v}
$$

where $\mathrm{G}$ is the shear modulus, a is the dot radius, $v$ is Poisson's ratio, and $\mathbf{u}$ is the displacement vector. Hence, a simple measurement of the displacement of the dot provides the force applied to the dot by the cell or platelet of interest. The mechanical properties of polyacrylamide are well characterized and can be tuned by simply changing the ratios of precursor materials [9].

Building on this concept, we have created a nanomechanical device which is able to measure the individual contraction of large numbers of platelets in a number of different biophysical and biochemical conditions (Figure 2). Platelets land, attach to, and contract pairs of protein dots which are patterned on a deformable poly-acrylamide (PAA) gel which is well characterized mechanically. The dot movement is linearly proportional to the applied contractile force [8], measured visually and analyzed using 
an image processing code written with MATLAB. By miniaturizing the gels into strips and surrounding them with a microfluidic, several different biochemical and biological conditions may be tested simultaneously. Furthermore, the microfluidic reduces the sample size and enables shear flow conditions, but more importantly limits the amount of time platelets spend in solution above the gel surface. All of this is achieved while being optically transparent and bio-compatible.

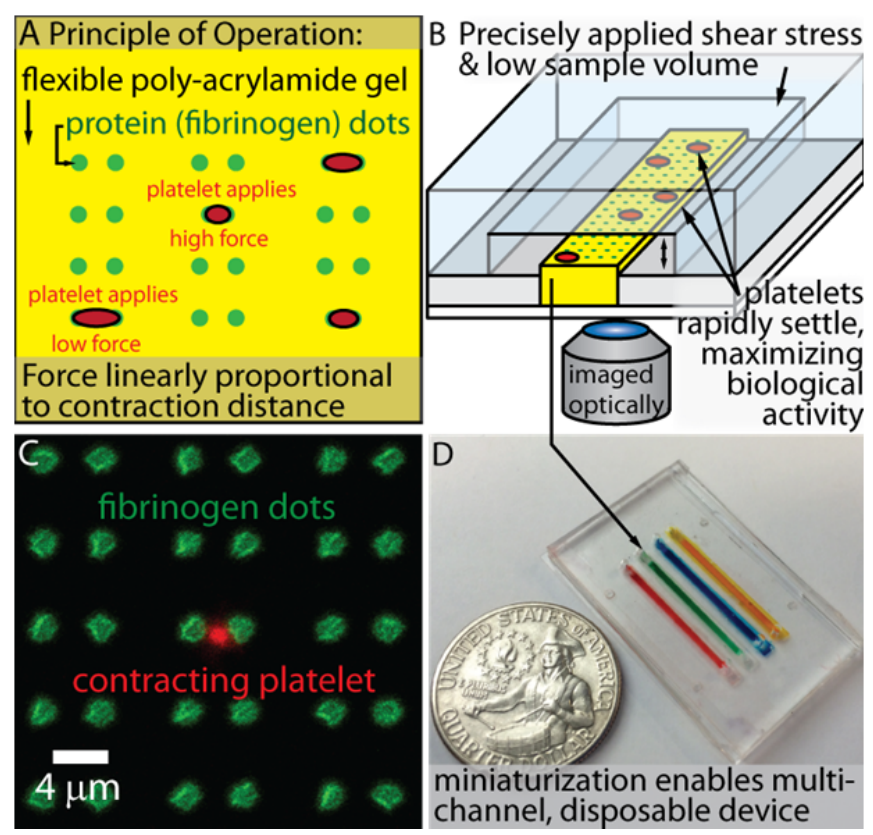

Figure 2: A) Platelets land, adhere, and contract the fibrinogen dots together. The applied force is linearly proportional to the contraction distance. B) Casting the gels in a strip and enclosing in a microfluidic creates the ability to precisely control applied shear stress, minimizes sample volume, and enables platelets to rapidly settle. C) Actual optical image of contracting platelet on fibrinogen dots. D) Actual device with four gel strips, each of different stiffness (and biochemical condition if desired).

In designing this system, it is important to note that platelets circulate through the bloodstream in a discoid, nominally inactive or resting state. When stimulated by certain agonists, such as thrombin or adenosine diphosphate (ADP), platelets experience immediate shape change, adhere to surfaces, and eventually clot. These agonists also upregulate integrins which assist in adhering to the protein dots on the gel (specifically $\alpha_{\mathrm{IIb}} \beta_{3}$ ). As such, platelets are loaded into the gels with agonist, but since they also begin undergoing shape change, one concern is that the platelet is expending energy which ultimately would be used towards actomyosin mediated contraction. By capitalizing on the unique ability of microsystems to minimize dimensions, platelets are on the gel surface in a matter of minutes rather than an hour. Once platelets reach the surface of the gel, they attach to a single dot, span to a neighboring dot, and contract. The force applied to the dots by the platelets will move them closer together, and the subsequent applied force can be calculated using equation 1 .

The sensitivity of the system is dependent on the optical resolution and the amount of displacement experienced by a protein dot for an applied force. The optical resolution in this system is dependent on how images are acquired. Typically, imaging is done from the bottom of the device. The coverslip is approximately $130 \mu \mathrm{m}$ thick, and the hydrogels are up to $400 \mu \mathrm{m}$ thick. As such, imaging was typically performed with a $20 x$
(0.8NA) objective with $488 \mathrm{~nm}$ light. In general, it was possible to resolve dot movements of $100 \mathrm{~nm}$ or greater. As such, it was important to maximize the movements of the dot for a given force. Previous measurements determined average force applied by platelets for a given simulated substrate stiffness [5]. Since the dot size and displacement are inversely proportional for a constant force, as shown in Eq 1, the dot size was minimized to maximize displacement. Since large arrays of dots were needed, the dot diameter was practically limited to $1 \mu \mathrm{m}$, which was the photolithographic limit of available tools. This design constraint does assume that the platelet contraction force is independent of the dot area. In extreme cases, this would be of concern since the protein dots themselves contribute to platelet activation and contraction through the $\alpha_{\mathrm{IIb}} \beta_{3}$ integrin.

The optimal dot spacing distance was determined from previous studies which examined the ability of platelets to span protein patterns on glass [10]. Here, it was important to balance the ability of platelets to span the gap between proteins with the range of forces which could be measured. Since the applied force is proportional to the dot distance, smaller gaps preclude the measurement of large forces. Here, a center to center distance of $4 \mu \mathrm{m}$ was found to enable platelets to span the protein dots but still provide an adequate range to measure all contracting platelets for stiffnesses used in this work.

\section{DEVICE FABRICATION \\ Laser Cut Gel Mold}

To create the microdevice, long rectangular holes $(1 \mathrm{~mm} x$ $25 \mathrm{~mm}$ ) were laser cut into a thin sheet of pre-fabricated PDMS. The PDMS sheet was cleaned with scotch tape and covalently bonded to a $24 \times 40 \mathrm{~mm}$ No. 1 coverslip after treatment with an $\mathrm{O} 2$ plasma for 30s. The initial bonding after bringing the coverslip and PDMS sheet together was weak and reversible, but is improved after heat treating at $60 \mathrm{C}$ overnight. The PDMS/coverslip piece was silanized by $\mathrm{O} 2$ plasma treating, and subsequently treated with an 10\%APTMES $/ 90 \%$ Ethanol $/ 0.01 \%$ Glacial acetic acid solution for $90 \mathrm{~min}$ at $60 \mathrm{C}$. The slides were removed and rinsed with $70 \% \mathrm{ETOH} / 30 \%$ DI water, then rinsed with DI water. The slides were then placed in DI water for 1 hour at room temperature. This water treatment significantly improved the PDMS flexibility and surface adhesion such that it could create a good seal when pressed against a second coverslip. Next, the slides were then treated with a $2 \%$ glutaraldehyde solution at room temperature for 30 minutes. The slides were then rinsed with DI water and dried with nitrogen.

\section{Fibrinogen Stamped Coverslip}

Stamped coverslips (No 1.5, 18mm x 18mm) were prepared using an etched silicon mold, previously described [11]. Briefly, AF488 fibrinogen was incubated on $10 \mathrm{~mm} \times 10 \mathrm{~mm} \times 3 \mathrm{~mm}$ PDMS squares at $30 \mathrm{ug} / \mathrm{mL}$ for 1 hour. A silicon mold, composed of an array of etched holes was ultrasonically cleaned in ethanol for 30 min, rinsed with DI water, dried, and treated with an $\mathrm{O}_{2}$ plasma for 30s. The PDMS squares were rinsed with DI water, air dried, and placed on the silicon mold. After adhering to the mold, the PDMS squares were removed and placed on $25 \mathrm{~mm}$ coverslips, which had previously been treated with an $\mathrm{O}_{2}$ plasma for 30 s. The silicon mold was preferred over using PDMS pillars to stamp the coverslip since the silicon based technique is capable of creating small fibrinogen features with large empty spaces. Then the PDMS squares were removed, leaving fibrinogen dots the $18 \mathrm{~mm} \times 18 \mathrm{~mm}$ coverslip.

\section{Mold Assembly}

The fibrinogen coverslip was then inverted over the PDMS/coverslip piece. The use of the $18 \mathrm{~mm} \times 18 \mathrm{~mm}$ coverslip with the long rectangular holes $(25 \mathrm{~mm})$, creates a channel with an 
enclosed center and open ends. The entire piece was placed in a $30 \mathrm{~mm} \mathrm{Hg}$ vacuum for 30 minutes just prior to placement into the argon glovebox. The vacuum helps remove some of the air from the PDMS, which interferes with gel polymerization. By stamping a large square of fibrinogen dots (15mm x $15 \mathrm{~mm})$, and using much smaller channels, some fibrinogen dots were always over the top of the polyacrylamide gel, easing alignment concerns.

Polyacrylamide Gel Preparation and Casting

A polyacrylamide solution with a ratio of acrylamide to bisacrylamide was created for each desired stiffness [9], with PBS. To assemble, a polyacrylamide solution, TMED, and APS, and NHSester were mixed in the glovebox directly prior to use. We specifically optimized this recipe to work in an argon glove box to reduce the amount of unpolymerized gel both along the edges of the coverslip and especially in the microdevice. To do so, the amount of TMED and APS typically used were reduced by 10 , and the working time was optimized to several minutes to ensure that the gels could be created in a rapid fashion.
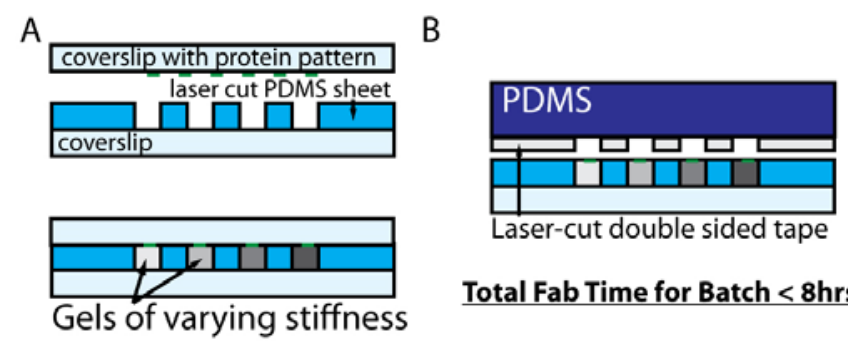

Total Fab Time for Batch $<8 \mathrm{hrs}$

Figure 3: Key fabrication steps: A) Protein is transferred from a stamped coverslip onto gels after the casting process. B) A microfluidic is adhered with a laser-cut double sided tape, leaving the protein intact

The components were kept separate until needed, since the gels will polymerize and since NHS-ester degrades in water. The liquid gel solution was then cast into the channels created by the coverslip and laser machined PDMS using a $20 \mu \mathrm{L}$ pipette. After polymerization ( $3 \mathrm{hrs}$ ) the gels were removed from the glovebox, and the top coverslip was discarded. Gels were then placed in PBS overnight.

\section{Microfluidic Top}

To cover the hydrogels, a separate SU-8 mold was fabricated, enabling the creation of PDMS microfluidic channels which are slightly larger than the hydrogel strips. Similarly, a piece of double sided tape was laser cut with rectangular holes matching that of the PDMS microchannel cover. After an overnight soak, the hydrogel chip was dried, with care taken to keep from displacing the hydrogels. Since PDMS sheet surrounding the hydrogels is hydrophobic, water tends to bead on the surface, facilitating removal with an aspirator. The double sided tape was then placed around the hydrogels, and the PDMS microchannels were then pressed onto the double sided tape. PBS was then flown into the enclosed channel until the device was ready for use. The double sided tape approach is unique in that it provides rapid attachment of a microfluidic without affecting the patterned proteins.

\section{Experiment}

Blood was drawn by median cubital venipuncture into acidcitrate-dextose (ACD) solution 2. Blood was spun at $150 \mathrm{G}$ for 15 minutes, platelet rich plasma was subsequently removed and further spun at $900 \mathrm{G}$. The resulting platelet pellet was resuspended in Tyrode's buffer with $0.1 \%$ bovine serum albumin. Platelets, $5 \mathrm{mM}$ of $\mathrm{CaCl}_{2}, 5 \mathrm{mM}$ of $\mathrm{MgCl}_{2}$, and agonist were added to the device and allowed to incubate for 2 hours. Platelets were then imaged using a Zeiss LSM 700-405 confocal microscope.

\section{Platelet Analysis}

Platelets were analyzed using a MATLAB script which measured the fibrinogen dot areas, and calculated the center to center distance of the fibrinogen dots. High pattern fidelity was achieved in creating the gels, but dots without platelets were also measured for comparison. Only platelets which spread over two dots and were free of significant filiopodia extending to other dots (in the case of thrombin related measurements) were counted. The platelet density was optimized to minimize platelet aggregates, and occasional platelet aggregates were not counted.

\section{RESULTS \& DISCUSSION}

This device has enabled the measurement of hundreds of platelets to date and has quantitatively measured the contraction force in a number of different biomechanical and biochemical conditions. The data is similar to the measurements taken by AFM, but with a much higher sample size. This specific distribution is not a normal distribution by Shapiro-Wilk at $\alpha=0.05$ (Figure 4).

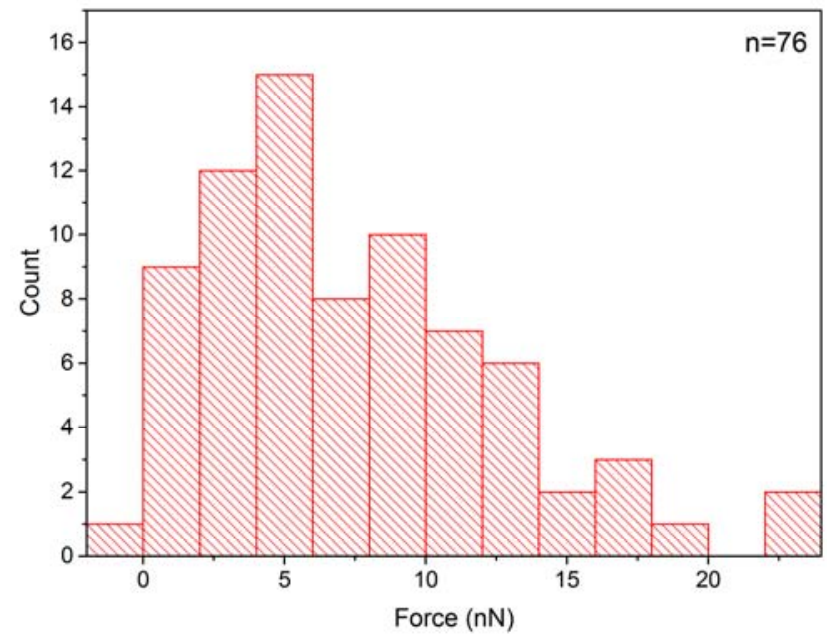

Figure 4. Distribution of platelet forces on a $25 \mathrm{kPa}$ gel with $1 \mathrm{U} / \mathrm{mL}$ of thrombin. The data agrees with previous measurements, albeit with a much higher sample number.

The casting process for any hydrogel stiffness between 0.5 and $100 \mathrm{kPa}$ is identical, enabling measurements of platelet response to the mechanical microenvironment with a single sample, removing uncertainty with day to day variations. This data shows that platelets are able to modulate the amount of force applied simply by changing the local microenvironment stiffness (Figure 5). Surprisingly, platelet contraction distances remain fairly constant among the different stiffness conditions, and are typically between 0.1 to $0.5 \mu \mathrm{m}$. This data also closely matches the previous AFM measurements [5], indicating that platelet contraction forces are generated independent of the exact surface used for measurement. The atomic force microscope experiments specifically used fibrinogen coated silicon nitride cantilevers, and the current experiments are using fibrinogen coated hydrogels.

These current experiments have also begun to quantify the exact differences in platelet contraction depending on the agonist used. Blood clots have been shown to be composed distinct regions, specifically a core of platelets activated by thrombin and a shell, activated by ADP [12]. The shell is composed of loosely attached platelets, permeable to $70 \mathrm{kD}$ dextran and eventually reduces in size. Here, it is shown that the contractile forces of platelets activated by ADP are significantly lower than that of 
thrombin, adding additional insight into how platelets can modulate their response depending on the local microenvironment.

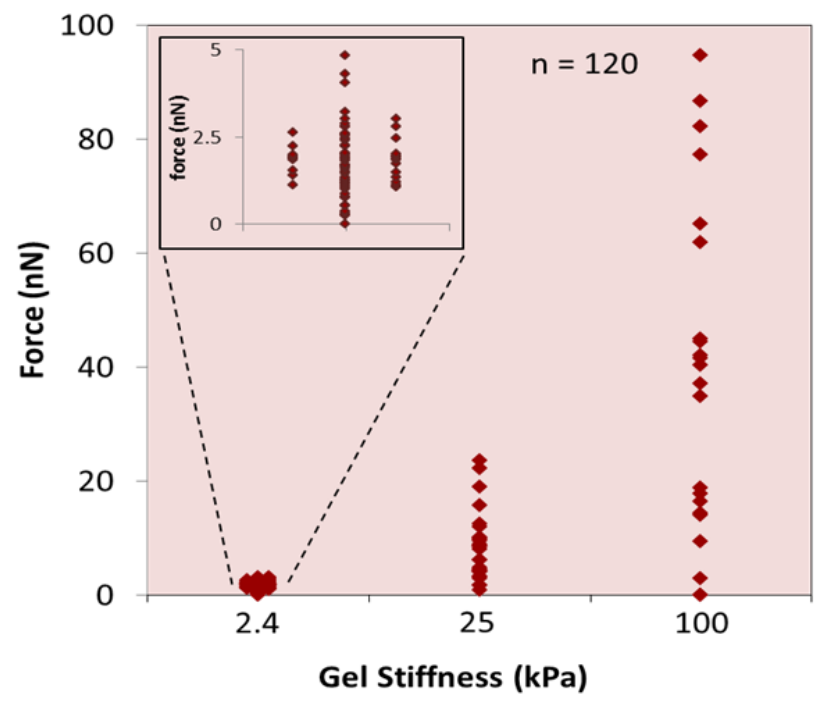

Figure 5: Platelets modulate the applied force based on the substrate stiffness. This data represents some of the most extensive gathered to date. The exerted forces are comparable to a muscle cell when corrected for size.

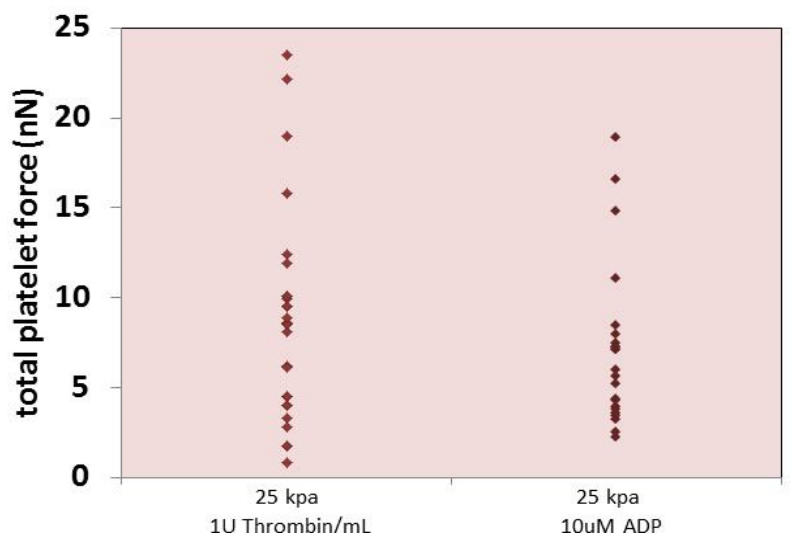

Figure 6: Different biochemicals alter the applied platelet force. Current hemostatic research indicates that a developing clot has two layers, a tightly bound inner layer, activated by thrombin, and an outer layer activated by ADP, which eventually peels off. Here we show that the different platelet activators also modulate the applied platelet force.

More importantly, this work establishes key metrics to identify healthy platelet contraction. By comparing clinical samples of patients with known bleeding disorders, it will now be possible to test the hypothesis that clot mechanics is directly affected by impaired or overactive platelet contraction.

\section{CONCLUSION}

Hydrogel casting integrates soft protein patterned surfaces with existing microtechnology, enabling a new class of precision device which more closely resembles the biological microenvironment. This is the first device to ever successfully measure large populations of platelets, with the data presented being the largest accumulated to date on individual platelet contraction. The device combines common microfabrication strategies with poly-acrylamide gel techniques (Figure 3) creating a rapid, disposable diagnostic device which measures real time platelet contraction. We have already characterized the contractile forces of hundreds of platelets, showing that the forces are normally distributed (Figure 4) and change under differing biophysical (Figure 5) and biochemical conditions (Figure 6) found in the body. This data will provide much needed information to inform clot contraction models as well as provide baseline data to characterize abnormal platelet function. This will lead to new diagnostics indicating increased risk for stroke or excessive bleeding, enabling proactive measures and potentially saving lives. Measurements of clinical samples are already underway.

\section{REFERENCES}

[1] J. W. Weisel, "Enigmas of Blood Clot Elasticity," Science, vol. 320, pp. 456-457, April 25, 20082008.

[2] J. P. Collet, Y. Allali, C. Lesty, M. L. Tanguy, J. Silvain, A. Ankri, et al., "Altered Fibrin Architecture Is Associated With Hypofibrinolysis and Premature Coronary Atherothrombosis," Arterioscler Thromb Vasc Biol, vol. 26, pp. 2567-2573, November 1, 20062006.

[3] A. Undas, A. Slowik, P. Wolkow, A. Szczudlik, and W. Tracz, "Fibrin clot properties in acute ischemic stroke: relation to neurological deficit," Thromb Res, vol. 125, pp. 357-61, 2010.

[4] A. Undas, K. Zawilska, M. Ciesla-Dul, A. LehmannKopydlowska, A. Skubiszak, K. Ciepluch, et al., "Altered fibrin clot structure/function in patients with idiopathic venous thromboembolism and in their relatives," Blood, vol. 114, pp. 4272-8. Epub 2009 Aug 18., Nov 52009.

[5] W. A. Lam, O. Chaudhuri, A. Crow, K. D. Webster, T.-D. Li, A. Kita, et al., "Mechanics and contraction dynamics of single platelets and implications for clot stiffening," Nat Mater, vol. 10, pp. 61-66, 2011.

[6] S. S. Henriques, R. Sandmann, A. Strate, and S. Köster, "Force field evolution during human blood platelet activation," Journal of cell science, 2012.

[7] X. M. Liang, S. J. Han, J.-A. Reems, D. Gao, and N. J. Sniadecki, "Platelet retraction force measurements using flexible post force sensors," Lab on a Chip, vol. 10, pp. 991998, 2010.

[8] S. Polio, K. Rothenberg, D. Stamenović, and M. Smith, "A micropatterning and image processing approach to simplify measurement of cellular traction forces," Acta biomaterialia, vol. 8, pp. 82-88, 2012.

[9] R. T. Justin and J. E. Adam, "Preparation of hydrogel substrates with tunable mechanical properties," Current protocols in cell biology, 2010.

[10] A. Kita, Y. Sakurai, D. R. Myers, R. Rounsevell, J. N. Huang, T. J. Seok, et al., "Microenvironmental Geometry Guides Platelet Adhesion and Spreading: A Quantitative Analysis at the Single Cell Level," PLoS ONE, vol. 6, p. e26437, 2011.

[11] A. C. von Philipsborn, S. Lang, A. Bernard, J. Loeschinger, C. David, D. Lehnert, et al., "Microcontact printing of axon guidance molecules for generation of graded patterns," Nature protocols, vol. 1, pp. 1322-1328, 2005.

[12] T. J. Stalker, E. A. Traxler, J. Wu, K. M. Wannemacher, S. L. Cermignano, R. Voronov, et al., "Hierarchical organization in the hemostatic response and its relationship to the plateletsignaling network," Blood, vol. 121, pp. 1875-1885, 2013.

\section{CONTACT}

*W.A. Lam, tel: +1-404-727-7473; wilbur.lam@emory.edu 